\title{
Evaluation of direct reading photoionization detector performance under various operational parameters
}

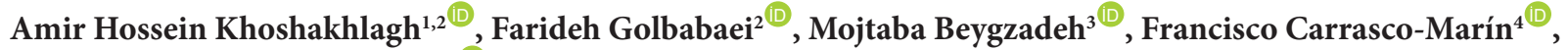 \\ Seyed Jamaleddin Shahtaheri ${ }^{2,5^{*}}$ \\ ${ }^{1}$ Department of Occupational Health Engineering, Faculty of Health, Kashan University of Medical Sciences, Kashan, Iran \\ ${ }^{2}$ Department of Occupational Health Engineering, Faculty of Public Health, Tehran University of Medical Sciences, Tehran, Iran \\ ${ }^{3}$ Department of Energy, Materials and Energy Research Center, Tehran, Iran \\ ${ }^{4}$ Carbon Materials Research Group, Faculty of Science, Avda. Fuente Nueva s/n, University of Granada, Spain \\ ${ }^{5}$ Institute for Environmental Research, Tehran University of Medical Sciences, Tehran, Iran
}

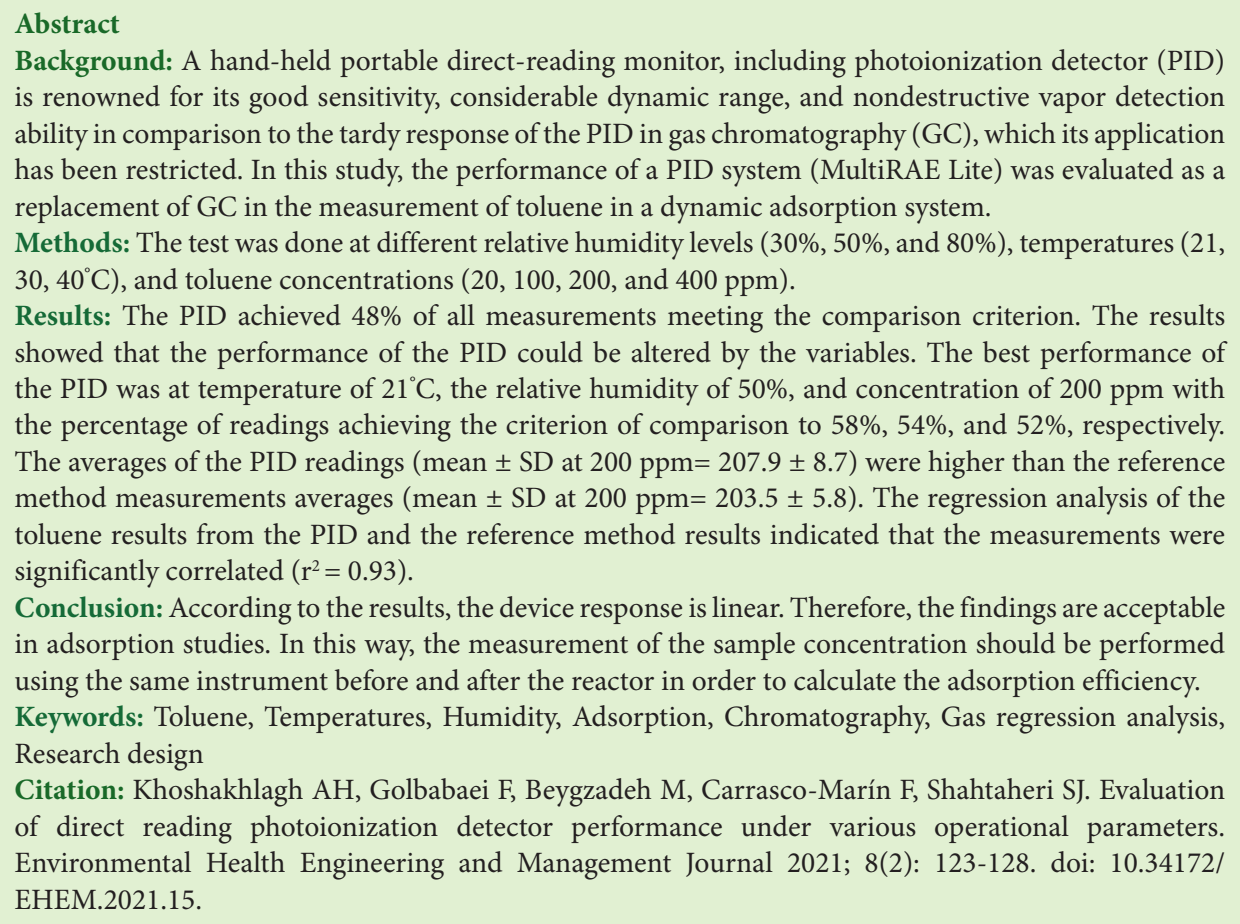

Article History:

Received: 15 December 2020 Accepted: 22 February 2021 ePublished: 14 May 2021

\section{Introduction}

According to the necessity of air pollution control, many studies have been done to adsorb, remove hazardous air pollutants, and evaluate effectively to control airborne gaseous pollutants (1-4). In this case, two groups of systems including static and dynamic systems are used. In a static system, the charcoal is put inside the reactor and a specified concentration of the pollutant, which is usually generated in an airbag, is passed through the reactor in a closed chamber $(1,4)$. For measuring the concentration of the pollutant in a closed system (static reactor), gas chromatography (GC) is commonly used (2).

In a dynamic system, the concentration of the sample, which is generated using a syringe pump, passes through the reactor with setting temperature and humidity. In this system, the adsorption or removal efficiency is reported as influent and effluent concentrations (1).

The concentration fluctuation in the dynamic system is a controversial issue $(1,5)$. The equipment for measuring the volatile organic compounds (VOCs) concentration is the GC equipped with the flame ionization detector (GC-FID) (1). GC has some advantages and limitations. The advantages of GC equipment are good accuracy and precision, high efficiency, and separation of compounds which can be vaporized without decomposition. Difficult access, required trained people, and large sample analysis 
costs are its limitations (6).

Time is very important to evaluate the adsorbent efficiency in the adsorption of pollutants through a dynamic system. The retention time for measuring the sample concentration in GC is almost high. The concentration fluctuations, which happen at the time of measuring the minimum or maximum adsorbent efficiency, will lead to losing some essential data about the adsorption capacity of the adsorbent. The mentioned limitations imply to use hand-held portable direct-reading monitors, including photoionization detector (PID) $(1,7)$.

The advantages of using a PID include a short-time period between the readings and providing the results, quick response, and recording fluctuations in the sample concentration. Also, it provides real-time exposure data in the field evaluation, and information in order to make informed decisions to control the environment. Furthermore, it can be used from the extracted data to choose the appropriate respiratory protection equipment $(5,8)$.

In a PID high-energy photons, mostly in the vacuum ultraviolet range, positively charged ions are produced by molecules. In the next step, the ions generate an electric current, which is shown as a signal on the detector. This electric current is displayed by a monitor that can be altered by changes in the amount of concentration. In other words, the higher the concentration of the sample, the more ions are generated, and the higher the current. Several parameters such as water vapor or temperature can affect the ions and lead to rearrangement and fragmentation of the ions (5,8-12).

The lamp in the PIDs plays an essential role in detecting pollutants. In this case, those ionization energies are similar to or lower than the energy of the photons generated by the PID lamp can just be detected or responded.

Toluene, which is one of the VOCs, is commonly used in adsorption studies. The ionization energy of toluene is about $8.81 \mathrm{eV}$. There are several PID lamps such as deuterium, krypton, and argon lamps with ionization energy of $10.2,1.06$, and $11.8 \mathrm{eV}$, respectively (10-12).

The reliability of the instrument is of the utmost importance. So identification of the accuracy and precision of the measurements along with bias must have been done. Accuracy is the degree to which the result of a calculation complies with the actual value or a standard. Precision is the refinement in measurement; in other words, it is the repeatability of the calculation. Bias is defined as the alteration between the average results of the calculated amount and the reference method (8).

Rismanchian et al conducted a study on the evaluation of a PID performance in photocatalytic studies. In this study, the sample had three xylene isomers, which was done at four concentration ranges and two humidity levels. The results of this study showed the difference between PID and GC measurements, which was in the range of 10-260 $\mathrm{ppm}$. In this study, all measurements obtained from the
PID were lower than the actual concentrations (11).

In another study by Coffey et al, the performance of four kinds of PIDs was studied. The findings showed that $42 \%$ of readings met the reference criterion $( \pm 25 \%)$. Readings from PIDs, including ppbRAE, IAQRAE, SapphIRe, MultiRAE, and TVA-PID groups were higher than the actual concentrations but those from TVA-FID group were lower than the actual concentrations (8).

Coy et al also compared the readings of the PID (RAE systems) with GC measurements. In this study, solvent mixtures (hydrocarbons) were studied. The findings showed that the PID readings underestimated the exposures in comparison with the GC measurements. The findings of this study revealed that the use of a PID was acceptable in order to assess exposures to solvent mixtures (9). Several studies on the evaluation of PIDs performance announced the linear responses for them $(1,5,11)$.

This study was conducted to evaluate the performance of a PID device in a dynamic system as a suitable alternative to GC equipment. The results of the PID were compared with a reference method in a condition that temperature, relative humidity $(\mathrm{RH})$, and concentration were varied to determine the effect of the above-mentioned parameters on the device performance.

\section{Materials and Methods \\ Instrumentation and calibration}

The measurements were performed using MultiRAE Lite with PID (model PGM-6208, RAE Systems, Inc.), equipped with a $10.6 \mathrm{eV}$ lamp. The PID was purchased for this research and was calibrated using the manufacturer's instruction before the beginning of the study. The calibration of the device was done in two ways, daily calibration using compressed zero air and span gas certified to contain $100 \mathrm{ppm}$ isobutylene in air.

\section{Operational parameters conditions}

In order to evaluate the effect of concentration on the PID performance, four concentrations of toluene ( $\geq 99.5 \% \mathrm{GC}$ grade toluene, Sigma Chemical Company, St. Louis, Mo.) $(20,100,200$, and $400 \mathrm{ppm})$ were used to challenge the PID performance. Toluene was chosen since it is a high production volume chemical. So, it is needed to sample this pollutant with direct-reading monitors (8). The four concentrations were chosen to be within the normal operational range of the monitors and also to include the National Institute for Occupational Safety and Health (NIOSH) recommended exposure limit (REL) and the Occupational Safety and Health Administration's (OSHA) permissible exposure limit (PEL) of $200 \mathrm{ppm}(2,5,7)$.

Three temperatures $\left(21,30\right.$, and $\left.40^{\circ} \mathrm{C}\right)$ and three relative humidity (RH) levels (30\%, 50\%, and $80 \%)$ were selected to challenge the PID performance since they encompass the PID operation limits of the and demonstrate conditions existed in industrial environments $(2,8,9)$. So, each trial was replicated four times over 36 experiments 
(four concentrations, three relative humidity levels, and three temperatures).

Six readings from the PID were made with the same interval for each experiment, and then, they were recorded.

\section{Experimental setup}

Figure 1 illustrates the experimental setup. The toluene vapor was produced by a syringe pump. Total airflow through the mixing chamber was $600 \mathrm{ml} / \mathrm{min}$, which was controlled by a flow meter. For each test, the appropriate temperature and relative humidity were controlled by electro-tech systems humidity and temperature controller and measured simultaneously under the same conditions. For each test, an Anasorb coconut shell charcoal tube (SKC Inc), which contained 2 parts (100 $\mathrm{mg}$ in the front and 50 $\mathrm{mg}$ in the back), was connected to a personal sampling pump (SKC, model 222; Eighty-Four, PA 15330 USA), which was set to a flow rate of $100 \mathrm{~mL} / \mathrm{min}$. Each sampling was taken 30 minutes. Before each experiment, the pump was calibrated using the electronic calibrator MesaLabs Bios Defender 520 UK, according to the manufacturer's instruction.

As shown in Figure 1, the particular toluene concentration was passed through 2 routes, one route for measuring toluene concentration according to the reference method, and the other for the PID value.

The NIOSH 1501 standard was used as a reference method (13). After sampling, the two sections of adsorbents were analyzed using FID-equipped Varian CP3800 GC.

Figure 2 shows the calibration curve of the toluene concentrations.

\section{Data analysis}

The average of the PID spontaneous readings was obtained in parts-per-million (ppm) concentration to compare with the reference method measurements results.

To represent that the averages of the PID readings and reference method measurements were equivalent, pairwise comparisons (one-way ANOVA) were performed.

The equivalency was calculated as the average of the PID readings within $\pm 25 \%$ of the average reference method measurements. This calculation was based on the Occupational Safety and Health Administration (OSHA) standard (8).

The test was done in two modes. One mode was performed for combined operational parameters (temperature, humidity, and concentration) and the other was done for the operational parameters, separately.

Linear regression analysis was performed using the reference method as a dependent variable and the PID as an independent variable.

\section{Results}

As mentioned in the previous section, four toluene concentrations were prepared using an experimental setup.
Operational parameters including three temperatures $\left(21 \%, 30 \%\right.$, and $\left.40^{\circ} \mathrm{C}\right)$ and three relative humidity levels $(30 \%, 50 \%$, and $80 \%)$ were determined for this study and each experiment was replicated four times.

The PID readings average had a $48 \%$ consistency with the reference method measurements average under combined operational parameters conditions. Figure 3

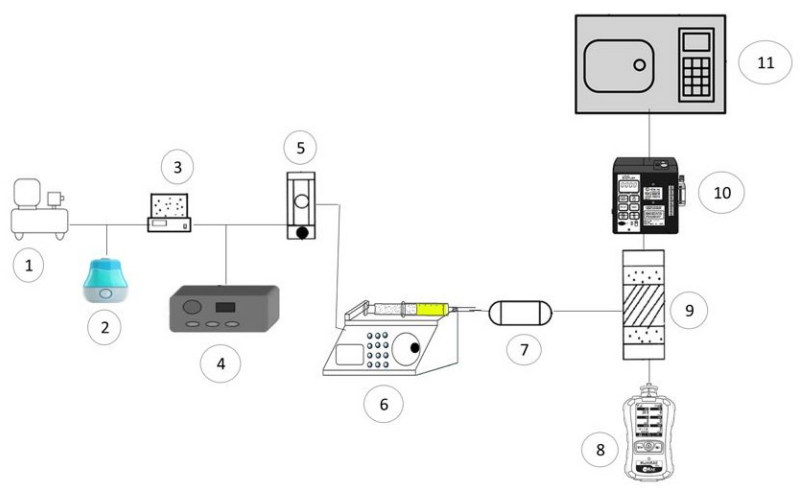

Figure 1. Schematic diagram of the experimental setup. 1) Air pump; 2) Humidifier; 3) Thermostatic water bath; 4) Humidity and temperature controller; 5) Flow meter; 6) Syringe pump; 7) Mixing chamber; 8) PID detector; 9) Fix-bed reactor; 10) Sample pump; 11) Gas chromatography.

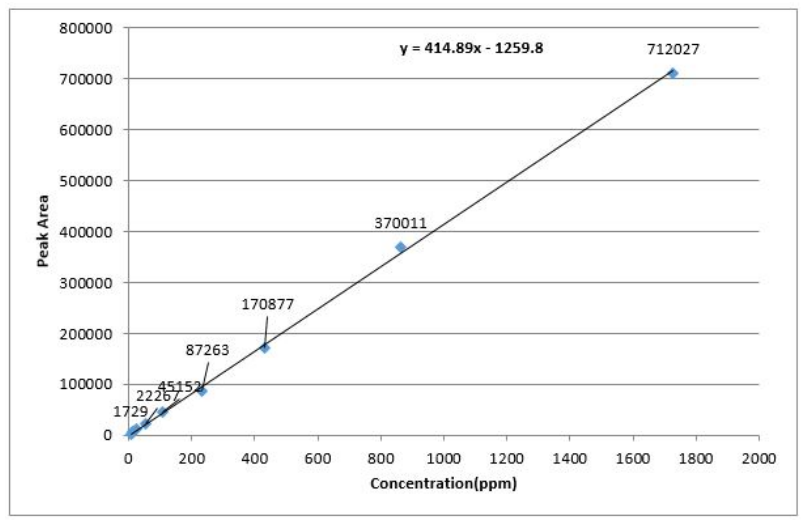

Figure 2. Gas chromatography calibration curve for the toluene concentrations $\left(r^{2}=0.999\right)$.

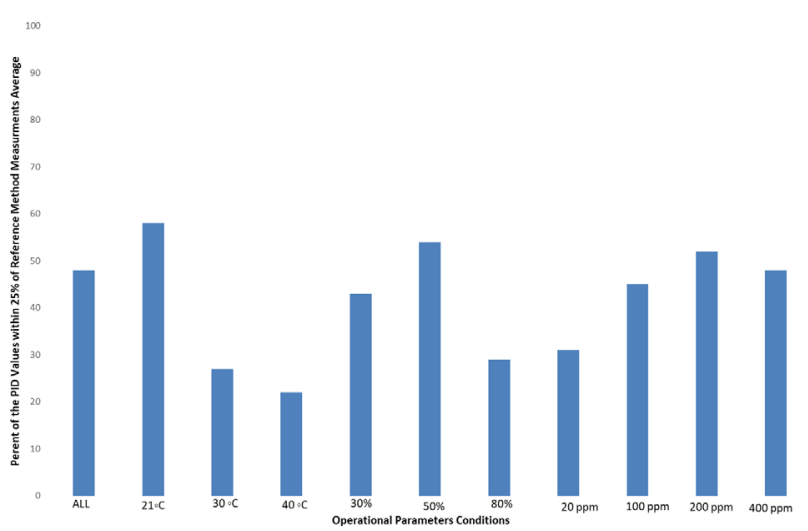

Figure 3. Pairwise comparison of the PID to the reference method using $25 \%$ criterion. 
illustrates the effect of temperature, relative humidity, and toluene concentration on the average of the reading of the PID compared to the average of the reference method measurements.

The highest comparability between the PID readings average and the reference method measurements average for the individual operational parameters conditions was found at temperature of $21^{\circ} \mathrm{C}$, relative humidity of $50 \%$, and toluene concentration of $200 \mathrm{ppm}$ with the percentage of $58 \mathrm{~T}, 54 \%$, and $52 \%$, respectively.

Table 1 presents the mean and standard deviation for the results of toluene measurement by the PID and reference method at different concentrations (ppm). The averages of the PID readings were higher than those of the reference method measurements. The PID indicated more significant variability, which is proved by their standard deviations.

The regression analysis of the toluene result from the PID by the reference method results indicated that the measurements were significantly correlated $\left(r^{2}=0.93\right)$ (Eq. 1).

$$
Y=4.473+1.024 X(1)
$$

Where $Y$ and $X$ values demonstrate the concentrations by the PID (ppm) and the actual concentrations (ppm), respectively.

The total mean of the instrument response factor for all concentration levels was about $1.09 \pm 0.02$, which means that the concentration of $20 \mathrm{ppm}$ demonstrated by the reference method is around the concentration of $29 \mathrm{ppm}$ by the PID.

\section{Discussion}

The data presented that the PID can be a reliable device for measuring the toluene concentrations using the dynamic system under different environmental conditions.

Although the PID data overestimated the actual concentrations, the high correlation with the reference method results showed that the PID response was representative of the actual measurements by the reference method. The findings demonstrated a linear relationship between actual measurements measured using the reference method and those measured by the PID. In the study by Rismanchian et al, a linear relationship between PID response and actual concentration values except the humidity level was reported, which is consistent with the results of the present study and the study of Coy et al, which showed a linear correlation between the PID responses and sample concentrations (9-11).

The PID showed the best performance at $21^{\circ} \mathrm{C}$. The best performance of toluene at $21^{\circ} \mathrm{C}$ can be due to this reason that the detection limit of the lamp of the PID is optimal at $21^{\circ} \mathrm{C}$ because of the inherent design of the device $(8,10)$. Coffey et al compared the effects of concentration, temperature, and humidity on the performance of several portable direct-reading organic vapor monitors (DROVMs). The sample was hexane. The best performance of the most DROVMs was reported at $21^{\circ} \mathrm{C}(8)$, which is consistent with the results of the present study.

The performance of the PID varied in response to concentration. The results indicated that the PID had a better performance at higher concentrations. The maximum response of the PID device was at toluene concentration of $200 \mathrm{ppm}$. The probable reason for the increase in the response of the PID direct reading device at toluene concentration of $200 \mathrm{ppm}$ could be due to the inherent design of the MultiRAE Lite device to detect the concentration of $200 \mathrm{ppm}(8,10)$. Rismanchian et al (11) evaluated the performance of a PID with a $10.6 \mathrm{eV}$ lamp in photocatalytic studies for removing VOCs. In their study, three xylene isomer mixtures were measured in air at three relative humidity levels $(0,20$, and $80 \%)$ and four concentration ranges (10-20, 50-60, 105-135, and 200-260 ppm). In this study, the coefficient of variation (CV) of the PID readings represents a decrease in the CV with increasing the reading concentration measurements. This can be due to more repeatable measurements at high concentrations. The results of the present study are consistent with those of other studies $(7,8,11)$.

Barsky et al reported that the response of a PID with a $10.8 \mathrm{eV}$ lamp was sensitive to the relative humidity so that the responses at $90 \%$ humidity in comparison to dry air for toluene was about $78 \%$. They found that water vapor decreased the performance of the PIDs (14). In the present study, the results for the PID varied across relative humidity levels, but no significant difference was found. The maximum response of the PID device was at $50 \%$ relative humidity, which can be due to rendering the detection of water vapor suitable at $50 \%$ relative humidity by the PID (10). The result of the present study is consistent with those of other studies $(8,11,14)$.

According to the regression equation, at the actual concentration of $20 \mathrm{ppm}$, the PID reading would be $25 \%$ high, and at $200 \mathrm{ppm}$, it would be $4.3 \%$ high.

The response higher than the expected PID response may be due to the existence of toluene as a representative

Table 1. Toluene measurement results by the PID and reference method at different concentrations

\begin{tabular}{lcccc}
\hline \multirow{2}{*}{ Measurements } & Mean \pm SD & Mean \pm SD & Mean \pm SD & Mean \pm SD \\
\cline { 2 - 5 } & $\mathbf{2 0 ~ p p m ~}$ & $\mathbf{1 0 0 ~ p p m ~}$ & $\mathbf{2 0 0} \mathbf{~ p p m ~}$ & $\mathbf{4 0 0} \mathbf{~ p p m ~}$ \\
\hline Reference method & $21.5 \pm 1.6$ & $93.6 \pm 6.3$ & $203.5 \pm 5.8$ & $406.7 \pm 4.1$ \\
PID & $26.3 \pm 8.1$ & $98.6 \pm 6.3$ & $207.9 \pm 8.7$ & $413.4 \pm 6.2$ \\
\hline
\end{tabular}


of VOCs with ionization potentials lower than $10.6 \mathrm{eV}$ in the test. The ionization potential of toluene is about 8.81 $\mathrm{eV}$.

Response factors can improve the correlations of the study of the PID (15-17). In a study by Drummond, the performance of a PID (MiniRae) was evaluated in the exposure of benzene. The obtained response factor was 0.2 for benzene (15). In another study by Rismanchian et al (11), the response factor for xylene mixture was calculated to be about 1.29 for a PID (PhoCheck) (11). In the present study, the response factor for the PID was calculated to be 1.09 , which means a $9 \%$ difference in the time of calibration of the instrument by standard gas. No significant difference was found in response factors at different humidity and temperature levels.

In this study, only one vapor (toluene) was tested. Therefore, investigating not a mixture of gases is the limitation of this study as few workplaces contain just a single gas or vapor.

The results of this study support this issue that, the PID should be calibrated to isobutylene to measure the toluene concentration as a representative of VOCs.

\section{Conclusion}

The PID results presented decisive determinations about the effects of temperature, relative humidity, and concentration on the device performance. The results also proved that the PID might not be reliable for precisely measuring concentrations of the sample at high humidity levels in a dynamic experimental set-up. The findings are acceptable in adsorption studies. Therefore, the measurement of the sample concentration should be performed using the same instrument before and after the reactor to calculate the adsorption efficiency. Calibration of the PID is necessary in order to obtain reliable data.

\section{Acknowledgments}

The authors would like to thank Tehran University of Medical Sciences and Iran National Science Foundation (INSF), for supporting this project. This study was supported by Tehran University of Medical Sciences under Ph.D. thesis scheme (Number: 9421138001) and the INSF. The funding body played no role in the design of the study, collection and analysis of data, interpreting results or writing the manuscript or decision to submit the manuscript for publication. The content of this publication is only the responsibility of the authors.

\section{Ethical issues}

This study was approved by the Research Ethics Committee of Tehran University of Medical Sciences (Ethical code: IR.TUMS.SPH.REC.1397.286). The authors certify that all data collected during the study are as presented in this manuscript, and no data from the study has been or will be published elsewhere separately.

\section{Competing interests}

Authors declared no conflict of interests.

\section{Authors' contributions}

AHK contributed to conception and design, execution and data collection. FG, MB, FSM, and SJSH contributed to data analysis and interpretation. All authors critically reviewed, refined, and approved the manuscript.

\section{References}

1. Bahri M, Haghighat F, Kazemian H, Rohani S. A comparative study on metal organic frameworks for indoor environment application: Adsorption evaluation. Chemical Engineering Journal 2017; 313: 711-23. doi: 10.1016/j. cej.2016.10.004.

2. Jahangiri M, Shahtaheri SJ, Adl J, Rashidi A, Kakooei H, Rahimi Forushani A, et al. The adsorption of benzene, toluene and xylenes (BTX) on the carbon nanostructures: the study of different parameters. Fresenius Environ Bull 2011; 20(4a): 1036-45.

3. Fooladi M, Moogouei R, Jozi SA, Golbabaei F, Tajadod G. phytoremediation of btex from indoor air by hyrcanian plants. Environ Health Eng Manag 2019; 6(4): 233-40. doi: 10.15171/EHEM.2019.26.

4. Talepour N, Hassanvand MS, Abbasi-Montazeri E, Latifi SM, Jaafarzadeh Haghighi Fard N, Shenavar B. Identification of airborne fungi's concentrations in indoor and outdoor air of municipal wastewater treatment plant. Environ Health Eng Manag 2020; 7(3): 143-50. doi: 10.34172/EHEM.2020.17.

5. LeBouf RF, Coffey CC. Effect of interferents on the performance of direct-reading organic vapor monitors. J Air Waste Manag Assoc 2015; 65(3): 261-9. doi: 10.1080/10962247.2014.986308.

6. McNair HM, Miller JM, Snow NH. Basic gas chromatography. 3rd ed. USA: John Wiley \& Sons; 2019.

7. Poirot P, Subra I, Gérardin F, Baudin V, Grossmann S, Héry M. Determination of short-term exposure with a direct reading photoionization detector. Ann Occup Hyg 2004; 48(1): 75-84. doi: 10.1093/annhyg/meg079.

8. Coffey CC, Pearce TA, Lawrence RB, Hudnall JB, Slaven JE, Martin Jr SB. Measurement capability of field portable organic vapor monitoring instruments under different experimental conditions. J Occup Environ Hyg 2008; 6(1): 1-8. doi: 10.1080/15459620802514728.

9. Coy JD, Bigelow PL, Buchan RM, Tessari JD, Parnell JO. Field evaluation of a portable photoionization detector for assessing exposure to solvent mixtures. AIHAJ 2000; 61(2): 268-74. doi: 10.1080/15298660008984536.

10. RAE. The PID Handbook-Theory and Applications of Direct-Reading Photoionization Detectors. 3rd ed. USA: RAE Systems Inc; 2013.

11. Rismanchian M, Golbabaei F, Mortazavi Y, Pourtaghi G, Rahimi Foroushani A. Evaluation of photoionization detector performance in photocatalytic studies for removing volatile organic compounds. International Journal of Environmental Health Engineering 2012; 1(1): 42. doi: 10.4103/2277-9183.102383.

12. Roithová J, Schröder D, Loos J, Schwarz H, Jankowiak HC, Berger R, et al. Revision of the second ionization energy of 
toluene. Journal of Chemical Physics 2005; 122(9): 094306. doi: 10.1063/1.1856916.

13. Azari MR, Konjin ZN, Zayeri F, Salehpour S, Seyedi MD. Occupational exposure of petroleum depot workers to BTEX compounds. Int J Occup Environ Med 2012; 3(1): $39-44$.

14. Barsky JB, Que Hee SS, Clark CS. An evaluation of the response of some portable, direct-reading $10.2 \mathrm{eV}$ and $11.8 \mathrm{eV}$ photoionization detectors, and a flame ionization gas chromatograph for organic vapors in high humidity atmospheres. Am Ind Hyg Assoc 1985; 46(1): 9-14. doi: $10.1080 / 15298668591394293$.

15. Drummond I. On-the-fly calibration of direct reading photoionization detectors. American Industrial Hygiene Association Journal 1997; 58(11): 820-2. doi: 10.1080/15428119791012333.

16. Soo JC, Lee EG, LeBouf RF, Kashon ML, Chisholm W, Harper M. Evaluation of a portable gas chromatograph with photoionization detector under variations of VOC concentration, temperature, and relative humidity. J Occup Environ Hyg 2018; 15(4): 351-60. doi: 10.1080/15459624.2018.1426860.

17. You DW, Seon YS, Jang Y, Bang J, Oh JS, Jung KW. A portable gas chromatograph for real-time monitoring of aromatic volatile organic compounds in air samples. J Chromatogr A 2020; 1625: 461267. doi: 10.1016/j.chroma.2020.461267. 